\title{
CORRECTED GSC: REFERENCE CATALOGUE FOR CCD OBSERVATIONS. NOW
}

\author{
A. LOPEZ GARCIA \\ Valencia University Observatory. Dept. Astronomy and \\ Astrophysics, Valencia, Spain. \\ AND \\ L.I.YAGUDIN \\ Pulkovo Observatory, Pulkovo, St.Petersburg, Russia.
}

\begin{abstract}
Astrometrical properties of the Hubble Space Telescope Guide Star Catalogue (GSC) were recently investigated by the same authors through its comparison with the PPM catalogue. In this paper, a new systematic plate-based magnitude dependent error produced by telescope optics and by the incompleteness of plate reduction model has been found after applying a simplified block adjustment procedure. New subroutine for correction of all systematic errors is developed and GSC can be used now as a dense reference catalogue on about 0.4 " accuracy level.
\end{abstract}

\section{Introduction}

Starting with the GSC authors (Russel at al., 1990) many astronomers were occupied by the GSC astrometric properties, trying to estimate the more evident systematic errors (Lasker at al., 1990; Taff at al., 1990; Bucciarelli at al., 1993; Roeser et al., 1994; Roeser et al., 1995), but nobody gave, up to now, a solution which could be used in astrometric practice immediately.

One year ago, we begun our investigations of the GSC properties with the aim of developing simple software which, connected with the Space Telescope Science Institute GSC CD ROM, could convert the GSC into a reference catalogue for narrow field instruments, representing the FK5 J2000 coordinate system (Lopez et al., 1994). Two systematic errors were found in the comparison of GSC with PPM catalogue: a plate correction due 
to the transformation into the FK5 system and a correction to be applied to each star according to its position on the GSC photographic plates (Lopez et al., 1995).

\section{GSC quasi-block-adjustment analysis}

GSC plate field corrections apply to GSC stars for the PPM magnitude range and it should be expected that GSC errors depend also on magnitudes, as faint stars errors have the same origin and nature as the bright stars ones, but on a different scale. Because of faint PPM stars absence, there is only one way to estimate the faint stars systematic errors, a significantly simplified block adjustment procedure applied to the overlapping parts of the GSC plates and based on the following assumptions:

1. Faint stars systematic errors are the same for all stars and all plates in narrow magnitude and declination zone intervals.

2. Faint stars systematic errors after applying the previous corrections have a very simple nearly radial form.

These assumptions are only approximated, but they simplify the procedure and give satisfactory results. To estimate the magnitude-dependent plate-based systematic errors, we have to solve by least squares method about 200 equation systems (for different declination zones and for different magnitude ranges) with a few number of unknown parameters in every system.

We choose the following nearly radial model for magnitude-dependent plate-based errors fitting:

$$
\begin{aligned}
& \delta_{x}=A * x * r+B * x * y^{2} \\
& \delta_{y}=C * y * r+D * y * x^{2}
\end{aligned}
$$

where $x, y$ are star standard coordinates on the GSC plate, $r$ is the star to plate center distance, $A, B, C, D$ are model parameters, and $\delta_{x}, \delta_{y}$ the errors under investigations.

First terms in both equations are the projections on the $x$ and $y$ axes of pure radial formula $\delta_{r}=k * r^{2}$ which can be considered as a plate radial distortion. Second terms are included to correct plate corner zones, as plate errors have 4 axes of symmetry.

Model parameters $A, B, C$ and $D$ were calculated separately for 6 magnitude ranges and 32 declination zones, corresponding to the GSC observational program.

In northern hemisphere, radial term dominates. It depends evidently on zenith distance and has physically clear magnitude dependence. Vice versa, in the southern sky, both terms are significant to the same extent 


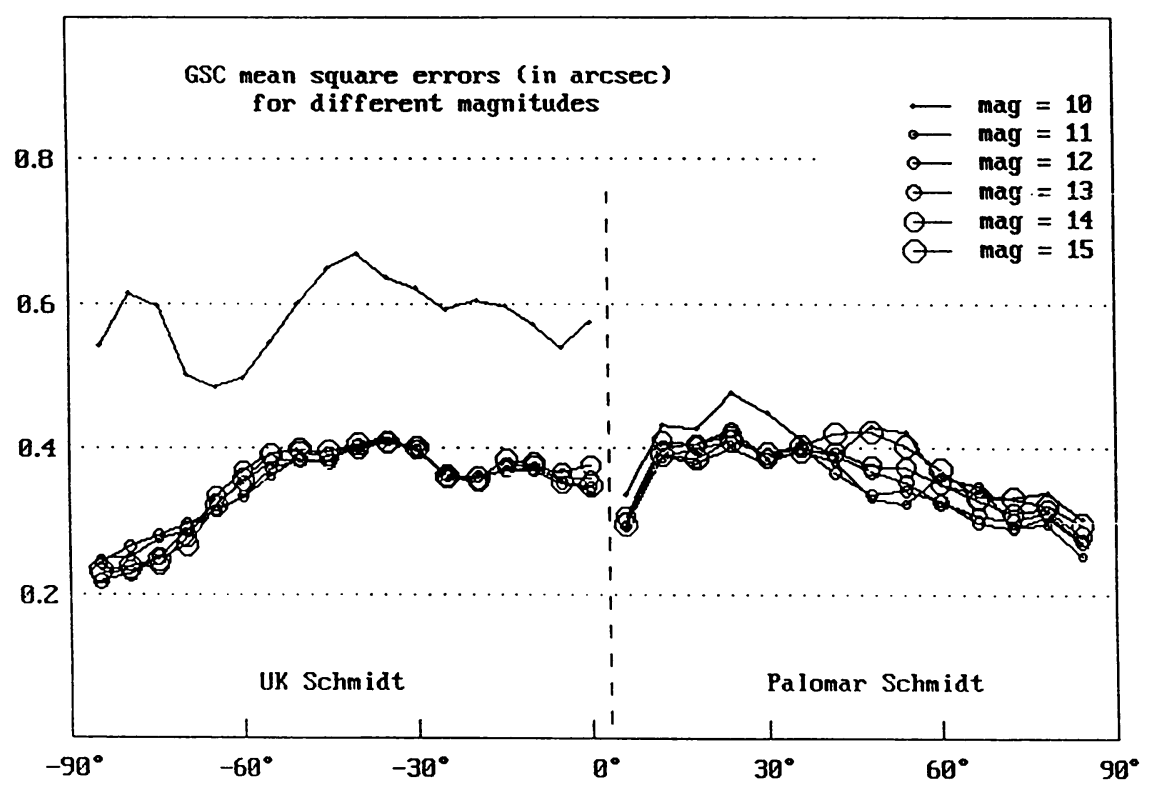

Figure 1. GSC accuracy

and their behavior is more complex. This means that GSC errors behavior is more sensitive to star brightness on the UK Schmidt plates than on the Palomar ones, as a result of the longer exposure time of the UK Schmidt plates (50-75 minutes) vs. 20 minutes for the Palomar plates. In all cases, the magnitude dependence is stronger for bright stars than for the faint ones, as it could be expected.

Systematic errors still remain in our model, but their values are significantly smaller than the source ones. According to our primary aim of investigating GSC systematic errors, we have included these results into our GSC correcting software as the third kind of correction.

\section{GSC accuracy}

Dispersion of the GSC-GSC' differences for the same stars from different plates after elimination of the three kinds of systematic errors gives an idea about the corrected GSC accuracy. Its mean values for different magnitudes and for different declination zones are shown on the Fig.1. As it is seen, the corrected GSC accuracy does not depend practically on star brightness for stars fainter than 10 magnitude; it depends on zone declination; it is practically the same for both telescopes. \pm 0.4 arcsec can be taken for the mean accuracy of the corrected GSC. It can be taken also for the upper estimation of the real GSC accuracy, which must be better because not all 
systematic errors are canceled by our corrections.

\section{Concluding remarks}

Our examination of the GSC is a very generalized one without taking into account the individual GSC plates properties and deeper investigations will give more information on the subject. Meanwhile, we hope that our simple software can be useful for those who need a really dense reference catalog, at least till appearance of the Hipparcos catalog or of a new Guide Star Catalog version.

\section{Acknowledgements}

The authors wish to thank the Conselleria de Ciencia y Educacion de la Generalitat Valenciana for financial support of this work.

\section{References}

Bucciarelli B., Lattanzi M.G., Taff L.G.: 1993, Ap. J. Suppl. 84, 91.

Lasker B.M., Sturch C.R., McLean B.J., Russel J.L., Jenkner H. and Shara M.M.: 1990, Astron.J. 99(6), 2019-2058.

Lopez Garcia A., Martinez Gonzalez J.M., Ortiz Gil A., Yagudin L.I.: 1994, IAU Comission 9, WG on "Wide - field imaging", Newsletter 6, 11-14.

Lopez Garcia A., Martinez Gonzalez J.M., Ortiz Gil A., Yagudin L.I.: 1995, Proc. of III International Workshop on Cel. Mech. and Posic. Astrom., Cuenca, Spain, 1994, in press.

Roeser S., Bastian U., Kuzmin A.: 1994 Proc. of IAU xxx, in press.

Roeser S., Morrison J.E. et al.: 1995 Astron.J., in press.

Russel J.L., Lasker B.M., McLean B.J., Sturch C.R. and Jenkner H.: 1990, Astron.J. 99(6), 2059-2081.

Taff L.G., Lattanzi M.G., Bucciarielli B., Gilmozzi R., McLean B.J., Jenkner H., Laidler V.G., Lasker B.M., Shara M.M. and Sturch C.R.: 1990, Astrophis.J. 353, L45-L48. 